\title{
QUASINILPOTENT OPERATORS AS NORM LIMITS OF NILPOTENT OPERATORS
}

\author{
BY E. H. LUCHINS AND K. C. MASTAN ${ }^{1}$ \\ Communicated by Bertram Yood, October 27, 1972
}

In [1, Problem 7], P. R. Halmos raised this problem: Is every quasinilpotent operator the norm limit of nilpotent operators? Since a limit of nilpotent operators may fail to be quasinilpotent, he noted that a better formulation of the problem would be: What is the closure of the set of nilpotent operators? In attempts at answers, details of which are in [4], [5], we studied $T$, an algebra of operators on $C[0,1][2, \mathrm{pp} .701-702] . T$ is radical and hence every element in it is quasinilpotent. Using \|\|$_{1}$, the sup norm, under which $T$ is a Banach algebra, we show that the closure of the set of nilpotent elements in $T$ is a maximal ideal. Hence any operator in $T$ not in this ideal is a quasinilpotent operator which, under the sup norm, is not the norm limit of nilpotent operators. However, we show that under an incomplete multiplicative norm for $T,\|\|_{2}$, every element in $T$ is the norm limit of nilpotent operators. Under both \|\|$_{1}$ and \|\|$_{2}$ there are sequences of nonnilpotent quasinilpotent operators in $T$ which converge to nonzero nilpotent operators.

There is a discontinuous isomorphism of the algebra $T$ under \|\|$_{2}$ onto $T$ under \|\|$_{1}$. Moreover, if there exists a complete multiplicative norm for $T$ under which every operator is the norm limit of nilpotent ones, then there exists a discontinuous isomorphism of $T$ under this norm onto $T$ under the sup norm. Thus the problem is related to the study of the continuity of automorphisms of nonsemisimple Banach algebras which wás urged by I. Kaplansky, who suggested that nontrivial radical algebras might be investigated $[3$, p. 3].

R. G. Douglas (cited in $[\mathbf{1}$, p. 916]) has shown that every compact quasinilpotent operator is the limit of nilpotent ones. The question of whether every quasinilpotent operator is either nilpotent or compact is raised and answered in the negative in [1, pp. 916, 918]. Our results, combined with Douglas' result, provide an alternative proof that there exist quasinilpotent operators which are neither nilpotent nor compact.

\section{REFERENCES}

1. P. R. Halmos, Ten problems in Hilbert space, Bull. Amer. Math. Soc. 76 (1970), 887-933. MR 42 \# 5066.

AMS (MOS) subject classifications (1970). Primary 46L20; Secondary 46J15.

${ }^{1}$ This note is based on a portion of a dissertation submitted by the second author to Rensselaer Polytechnic Institute in partial fulfillment of the requirements of the Ph.D. degree. 
2. E. Hille and R. S. Phillips, Functional analysis and semi-groups, rev. ed., Amer. Math. Soc. Colloq. Publ., vol. 31, Amer. Math. Soc., Providence, R.I., 1957. MR 19, 664.

3. I. Kaplansky, Algebraic and analytic aspects of operator algebras, CBMS Regional Conference Series in Math., vol. 1, Amer. Math. Soc., Providence, R.I., 1970.

4. Kathleen C. Mastan, On properties of radical Banach algebras, Doctoral Thesis, Rensselaer Polytechnic Institute, Troy, New York, 1972.

5. Kathleen C. Mastan and Edith H. Luchins, Quasinilpotent and nilpotent elements (in preparation).

Department of Mathematics, Rensselaer Polytechnic Institute, Troy, New York 12181 